Check for updates

Cite this: RSC Adv., 2019, 9, 24241

\title{
Thermosensitive double network of zwitterionic polymers for controlled mechanical strength of hydrogels $\uparrow$
}

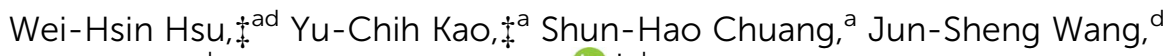 \\ Juin-Yih Lai ${ }^{\text {abc }}$ and Hsieh-Chih Tsai (iD *ab
}

Zwitterionic hydrogels have promising potential as a result of their anti-fouling and biocompatible properties, but they have recently also gained further attention due to their controllable stimuli responses. We successfully synthesized two zwitterionic polymers, poly(2-methacryloyloxyethyl phosphorylcholine) (poly-MPC) and poly(2-(methacryloyloxy)ethyl dimethyl-(3-sulfopropyl)ammonium hydroxide) (poly-DMAPS), which have complementary ionic sequences in their respective zwitterionic side groups and likely form an interpenetrating double network to improve their mechanical strength. The synthesized poly-MPC was blended in a poly-DMAPS matrix (MD gel) and showed high viscosity, while poly-DMAPS was blended in a poly-MPC hydrogel (DM gel) and revealed UCST behavior as the temperature increased. In addition, cross-section images of the MD hydrogel exhibited its compact and uniform structure, while the DM gel was found to exhibit a porous micro-structure with clear boundaries. The results explained the low viscosity of the DM gel, which was also confirmed via 3D Raman mapping. To sum up, the preliminary data demonstrated that binary zwitterionic hydrogels have thermosensitive mechanical properties, promoting further bio-applications in the future, such as in

wound healing.

Received 21st May 2019

Accepted 9th July 2019

DOI: $10.1039 / c 9 r a 03834 \mathrm{e}$

rsc.li/rsc-advances

\section{Introduction}

Synthesized hydrophilic polymers can offer wide-ranging designs for gelation via physical or chemical cross-linking, resulting in the construction of three-dimensional polymer network structures and good water swelling properties. ${ }^{1}$ Of these, in particular, zwitterionic polymers forming hydrogels show anti-fouling properties and have indirect pro-healing effects for use in implantable medical devices, tissue scaffolds and, especially, wound care..$^{2-4}$ However, hydrogels composed of chemically crosslinked polyzwitterions have issues with respect to their mechanical limitations. ${ }^{5}$ In the literatures, several methods have been used to improve the mechanical strength of

\footnotetext{
${ }^{a}$ Graduate Institute of Applied Science and Technology, National Taiwan University of Science and Technology, Taiwan, Republic of China. E-mail: h.c.tsai@mail.ntust.edu. tw

${ }^{b}$ Advanced Membrane Materials Center, National Taiwan University of Science and Technology, Taiwan, Republic of China

${ }^{C} R \& D$ Center for Membrane Technology, Chung Yuan Christian University, Taoyuan, Taiwan, Republic of China

${ }^{d}$ Instrument Technology Research Center, National Applied Research Laboratories, Taiwan, Republic of China

$\dagger$ Electronic supplementary information (ESI) available: The materials and methods and further data supporting these studies are provided as a separate document in the supplementary information. See DOI: 10.1039/c9ra03834e $\ddagger$ The authors equally contributed to this work.
}

hydrogels, such as the formation of non-covalent bond (ionic interactions ${ }^{6}$ and hydrogen bonds $s^{7}$ ) hydrogels with highly stretchable networks, ${ }^{8}$ and double-network (DN) hydrogels. ${ }^{9-11}$ The way in which DN hydrogels improve mechanical strength is through the two polymer matrices, as one can provide a rigid but brittle network and the other can present a soft and ductile matrix, preventing crack propagation and resisting hydrogel breaks. ${ }^{11,12}$ The most typical DN gel pairs consist of poly(2acrylamido-2-methylpropanesulfonic acid) gel and polyacrylamide gel, with upgraded strength 100-1000 times higher than the individual gels..$^{10,11,13}$ The difference here is that we do not prepare an ultra-high toughness DN hydrogel in this study but design an excellent anti-biofouling hydrogel with controlled mechanical strength using a dual-polyzwitterionic double network. Two polyzwitterions are individually prepared via the polymerization of either 2-methacryloyloxyethyl phosphorylcholine (MPC) or [2-(methacryloyloxy) ethyl]dimethyl-(3-sulfopropyl)ammonium hydroxide (DMAPS). There are complementary ionic sequences on the zwitterionic side groups of MPC and DMAPS, likely providing potential interpenetration interaction sites via coulombic interactions. ${ }^{10,14,15}$ Poly-MPC is composed of a cell-membrane structure with phosphorylcholine as a side chain and it has hemocompatible properties and cell-adhesion resistance, ${ }^{16-18}$ while poly-DMAPS is a well-known zwitterionic polymer having upper critical solution temperature (UCST) behavior. ${ }^{19,20}$ When the temperature is lower than the 
UCST, poly-DMAPS chains aggregate, enhancing chain entanglements and exhibiting higher viscosity. ${ }^{19,20}$ Once above the UCST, the polymers preferably change to the sol state, relieving the polymer tension and reducing the viscosity of the hydrogel. ${ }^{21}$ Herein, we expect that this dual-polyzwitterionic matrix could not only bolster the mechanical strength of a bulk-type hydrogel but it could also lead to control of the thermoresponsive mechanical properties for controlled sustained release. In future applications, such as wound healing care, thermosensitive hydrogels have promising potential to encapsulate painkiller medicines in wound patches and thus accelerate drug release via a warming compress, promptly achieving pain relief.

\section{Results and discussion}

The components of the synthetic zwitterionic polymers and the formation of truly independent-DN gels (no covalent bonds between the two polymeric networks) are depicted in Fig. 1. The synthesis processes can be divided into two parts: one involves homo-polymer synthesis and the other involves the prepared homo-polymers being mixed in secondary crosslinked matrices to form truly independent DN gels. ${ }^{10}$ The combination of polyMPC and poly-DMAPS to improve the intermolecular interactions forms the basis of the thermosensitive mechanical strength of the hydrogels in this study.

Firstly, zwitterionic homopolymers (either poly-MPC or polyDMAPS) were synthesized via UV irradiated free radical polymerization and characterized via ${ }^{1} \mathrm{H}-\mathrm{NMR}$. The proton peaks corresponding to the side chains of poly-MPC are located at $\delta 3.8 \mathrm{ppm}\left(-\mathrm{CH}_{2} \mathrm{~N}^{+}-\right), 4.1 \mathrm{ppm}\left(-\mathrm{POCH}_{2} \mathrm{CH}_{2} \mathrm{O}-\right)$, and $4.3 \mathrm{ppm}$ $\left(-\mathrm{NCH}_{2} \mathrm{CH}_{2} \mathrm{OP}-\right)$. On the other hand, the peaks from the polyDMAPS chains appear at $2.2 \mathrm{ppm}\left(-\mathrm{NCH}_{2} \mathrm{CH}_{2} \mathrm{CH}_{2} \mathrm{SO}_{3}\right)$ and $3.0 \mathrm{ppm}\left(-\mathrm{CH}_{2} \mathrm{CH}_{2} \mathrm{SO}_{3}\right)$ in the ${ }^{1} \mathrm{H}-\mathrm{NMR}$ spectrum (ESI, Fig. $\mathrm{S} 2 \dagger$ ). In addition, the double peaks from vinyl groups (at 5.8 and 6.3 ppm) disappear, indicating that the radical polymerization reaction has been completed. Following the structural characterization, it is practical to tune the critical temperature around the physiological temperature range; for this, the molecular weight and concentration of the polymers are two pivotal parameters to control the thermal response. The long polyDMAPS chains preferably form hydrophobic associations, leading to precipitation in aqueous solvent. On the other hand, the low molecular weight of the polymer makes it difficult to form such associations between the polymer chains. ${ }^{19}$ Herein, we adjusted the ratio of photo-initiator to monomer to different values $(0.5 \mathrm{~mol} \%$ and $1 \mathrm{~mol} \%)$ to prepare poly-DMAPS with two molecular weights $\left(M_{\mathrm{n}} 21 \mathrm{k} \mathrm{Da}\right.$ and $\left.46 \mathrm{k} \mathrm{Da}\right)$, which were characterized by GPC as shown in Fig. S3. $\dagger$

In order to develop a hydrogel with UCST functionality, the USCT properties of the poly-DMAPS should be well understood. Turbidity measurements are a simple and common method to evaluate UCST behavior. The UCST transition temperature is defined as the temperature at the cloud point, which is taken as the $50 \%$ transmittance of visible light in UV-visible absorption spectra. ${ }^{22}$ When the solution temperature is below the transition temperature, the polymers aggregate and present a cloudy

(a)<smiles>C=C(C)C(=O)OCCOP(=O)([O-])OCC[N+](C)(C)C</smiles>

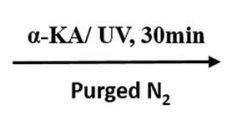<smiles>COC1OC(C)C(C(C)(C)C)C1(C)C</smiles><smiles>CCOP(=O)([O-])O</smiles>

Poly-MPC

(b)<smiles>C=C(C)C(=O)OCC[N+](C)(C)CCCS(=O)(=O)[O-]</smiles><smiles>[Mg][Mg][Mg]</smiles><smiles>COC(=O)C(C)(C)C(C)(C)C</smiles><smiles>CCC[N+](C)(C)CCCS(=O)(=O)[O-]</smiles>
Poly-DMAPS

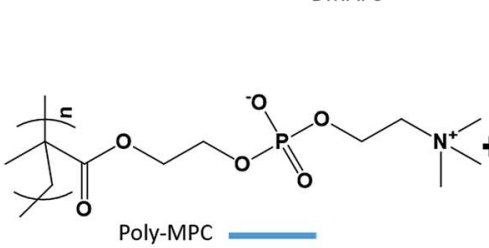<smiles>C=C(C)C(=O)OCC[N+](C)(C)CCCS(=O)(=O)[O-]</smiles><smiles>C=CC(=O)NCNC(=O)C=C</smiles>
$\mathrm{N}, \mathrm{N}^{\prime}$-Methylenebis(acrylamide)

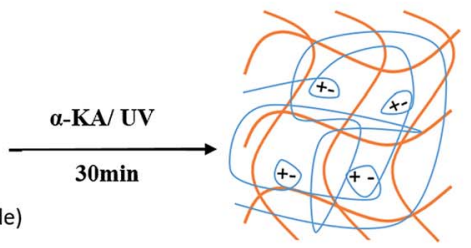
MD gel<smiles>CC(C)(C)C(C)(C)C(=O)OCC[N+](C)(C)CCCS(=O)(=O)[O-]</smiles><smiles>C=C([CH2+])C(=O)OCCOP(=O)(O)OCC[N+](C)(C)C</smiles><smiles>C=CC(=O)NCNC(=O)C=C</smiles>
$\mathrm{N}, \mathrm{N}^{\prime}$-Methylenebis(acrylamide)

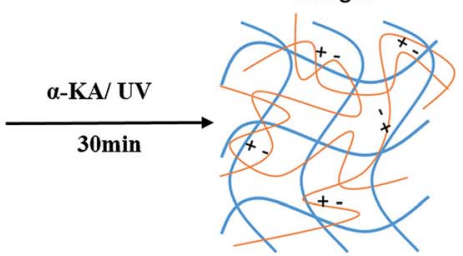

DM gel

Fig. 1 A schematic illustration of polymerization and double network hydrogel formation. (a) The preparation of the initial polymers. (b) Polymerization through mixing the initial polymers (either poly-MPC or poly-DMAPS) with the respective secondary monomers and a crosslinker to form double network hydrogels. Note: MD gel represents the initial polymer poly-MPC mixed in chemically crosslinked poly-DMAPS hydrogel. In contrast, DM gel represents the initial polymer poly-DMAPS mixed in chemically crosslinked poly-MPC hydrogel. 
appearance in solution. On the contrary, the aggregated polymer chains untangle and the solution turns transparent once the temperature becomes higher than the UCST. During turbidity measurements (Fig. 2), the low molecular weight polyDMAPS shows a relatively low cloud point at three concentrations, compared to the high molecular weight polymer. The short polymer segments may cause aggregation between polymer chains, but only a small amount of heat is required for dissolution. ${ }^{23}$ In addition, the different polymer concentrations presented different turbidity profiles. When the concentration of low molecular weight poly-DMAPS increased from $1 \mathrm{wt} \%$ to $10 \mathrm{wt} \%$, the cloud point shifted from $35{ }^{\circ} \mathrm{C}$ to $51{ }^{\circ} \mathrm{C}$, while the high molecular weight polymer presented a high UCST above $50{ }^{\circ} \mathrm{C}$ at all concentrations. As the result, the low molecular weight $\left(M_{\mathrm{n}} 21 \mathrm{k} \mathrm{Da}\right)$ homopolymer poly-DMAPS was chosen for the following studies because its UCST range ( $1 \mathrm{wt} \%$ to $10 \mathrm{wt} \%$ ) covered the physiological temperature range. Although the cloud point of lower molecular weight $\left(M_{\mathrm{n}} \leq 20 \mathrm{k} \mathrm{Da}\right)$ polyDMAPS was also measured via turbidity testing, the polyDMAPS chains are too short to aggregate, even at room temperature.

Following the preparation of the initial zwitterionic homopolymers (either poly-MPC or poly-DMAPS), one prepared polymer was mixed with the other monomer and a crosslinker before polymerization to form bulk hydrogels in home-made cells $(18 \mathrm{~mm} \times$ $18 \mathrm{~mm} \times 5 \mathrm{~mm}$, Fig. S1 $\dagger$ ). In this study, there are two DN gel prototypes, as shown in Fig. 1. One is the synthesized homopolymer poly-MPC mixed in a crosslinked poly-DMAPS hydrogel, which is called MD gel. The other is poly-DMAPS immersed in a crosslinked poly-MPC hydrogel (DM gel), as shown in Fig. S4. $\dagger$ We expect that both gels will have different characteristics due to the thermally responsive poly-DMAPS existing in different matrices, either incorporated as linear polymers unbound in a crosslinked matrix or bound in a chemically crosslinked network; therefore it will likely present varying UCST behavior in the hydrogels.

For the characterization of bulk-type samples, Raman spectroscopy is a useful and direct method and it takes advantage of low sensitivity to hydrated samples. ${ }^{24}$ It can provide a quick characterization of bulk gels to obtain the composition ratio and homogeneity without breaking samples. In Fig. 3, the interactions between incident photons and different functional groups of poly-MPC and poly-DMAPS have been qualified and quantified. The peaks at $795 \mathrm{~cm}^{-1}$ correspond to the phosphate group of poly-MPC (labelled as peak "p") ${ }^{25}$ and the peaks at $1034 \mathrm{~cm}^{-1}$ are attributed to the sulfonate group of poly-DMAPS (labelled as peak "s"). ${ }^{26}$ In addition, the composition of the bulk hydrogels was firstly determined from the intensity ratios of the selected peaks. The spectra can present the intensity ratios of the selected functional groups at different monomer concentrations $\left(C_{[\mathrm{MPC}]}: C_{[\mathrm{DMAPS}]}\right)$. However, an increasing concentration of the DMAPS monomer being present during polymerization did not result in a proportional composition in the hydrogel. Herein, a reasonable explanation is that it is like that MPC has been diluted by DMAPS monomers. Moving from 1D Raman spectra to 2D Raman mapping, we chose the "p" and "s" peaks to investigate the distribution of the two polymers in a cross-section of MD gel. From this we can see that MD gel presents a clearly homogeneous distribution of both polymers, as shown in Fig. 4a.

Furthermore, bulk hydrogels (MD gel, DM gel, random gel) were characterized via 3D-mapping Raman scanning to investigate the structural homogeneity. ${ }^{27}$ Based on the selected peak of the sulfonate group of poly-DMAPS, tomographic Raman mapping was performed from the intensity distribution in a $3 \mathrm{D}$ micro-zone $\left(10 \times 10 \times 10 \mu \mathrm{m}^{3}\right)$. A high Raman intensity indicated potential scenarios, such as self-chain interactions, which are likely caused by UCST-related aggregation. More interestingly, $X-Z$ cross-section images of the DM gel showed noticeable differences in intensity signals, as shown in Fig. 4c, demonstrating more inhomogeneity in this dual-polymeric matrix compared to MD gel. The phase separation of the DM gel is likely caused because thermosensitive poly-DMAPS preferably demonstrates intramolecular and intermolecular attractions between the DMAPS side chains at temperatures below the UCST.

In addition, the $X-Z$ cross-section mapping images of a random gel as a control presented the slight aggregation of either poly-MPC- or poly-DMAPS-dominated phases, distributed

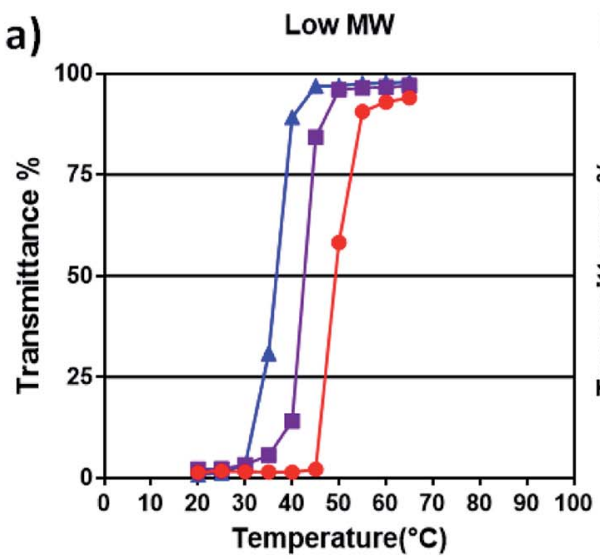

b)

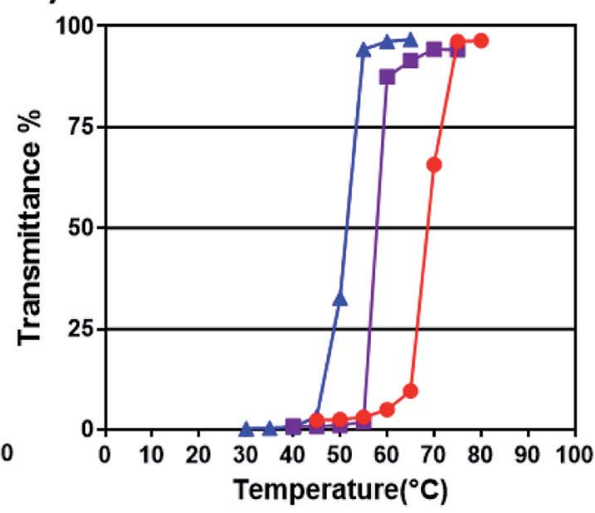

c)

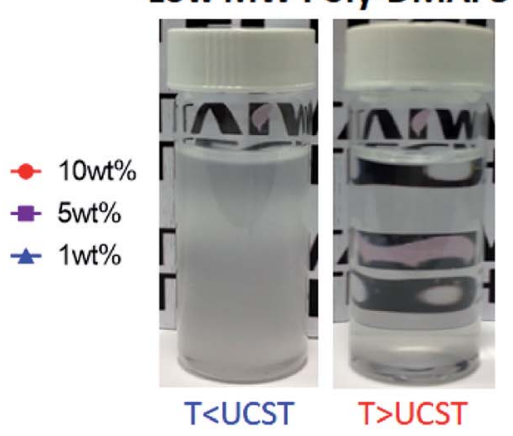

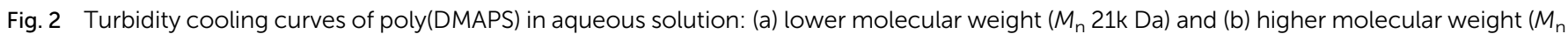

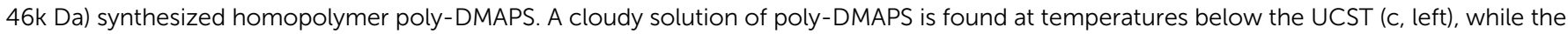
solution becomes transparent at temperatures above the UCST (c, right). 
Ratio 1:1

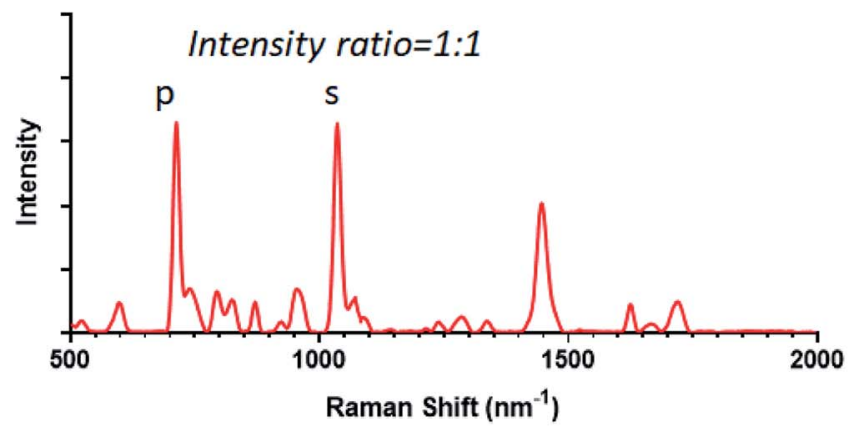

Ratio 1:3

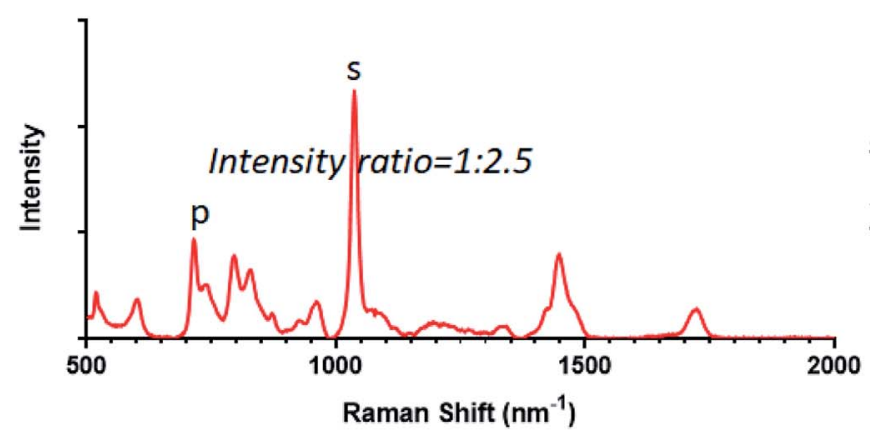

Ratio 1:2

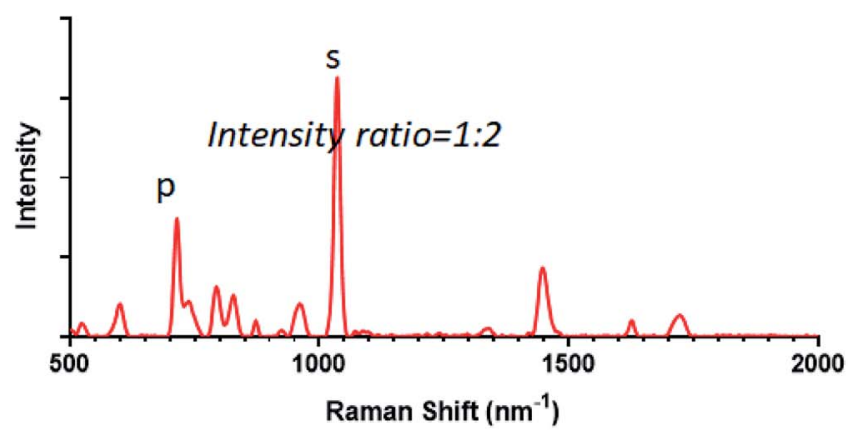

Ratio 1:4

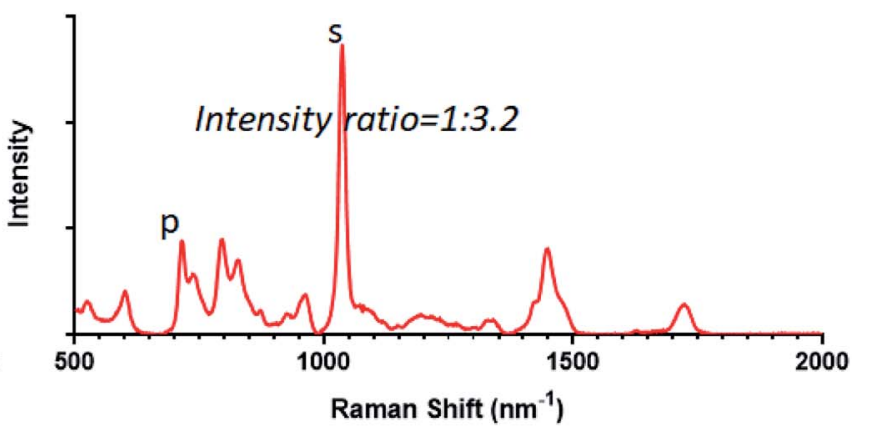

Fig. 3 Raman spectra analysis of hydrogels formed via the random polymerization of various monomer concentration ratios $\left(C_{[M P C]}: C_{[D M A P S]}=\right.$ $1: 1$ to $1: 4)$. The peaks marked " $p$ " represent the phosphate groups of poly-MPC chains and the peaks marked "s" represent the sulfonate groups of poly-DMAPS chain.

in a random pattern through the gel (Fig. 4d). Since the random gel was prepared via the one-step polymerization of DMAPS and MPC monomers with a chemical crosslinker, the zwitterionic side chains of both monomers undergo interactions with each other before polymerization. As the result, there is no inherent UCST behavior between the polymer chains, because once polymer chains are bound by the chemically crosslinked matrix, the UCST polymers do not have enough freedom to undergo sufficient intra-molecular interactions and the MPC side chains can even interfere with the micro-structural aggregation of polyDMAPS segments during polymerization.

To study the double network rheology and thermosensitive response of the zwitterionic hydrogels, the viscosities of the DM gel, MD gel and random gel were measured; they are ranked in the following sequence: MD gel $>$ random gel $>$ DM gel $>$ polyDMAPS-only gel $>$ poly-MPC-only gel, at slow shear rates $\left(10^{-3}\right.$ to $10^{-2} \mathrm{~s}^{-1}$ ), as shown in Fig. 5a. All two-component hydrogels (MD gel, DM gel, random gel) were found to exhibit higher viscosities than the single-component hydrogels made from either poly-DMAPS or poly-MPC, proving the concept that the double network boosts the hydrogel mechanical properties. This concept was similarly confirmed through mixing the linear polyzwitterions in the same polyzwitterionic chemical networks. ${ }^{28}$

In our binary polyzwitterion hydrogel systems, the properties of the highest strength gel, the MD gel, were attributed to both the physical and chemical crosslinked networks, which cofacilitated the formation of a uniform double network structure. ${ }^{14,15}$ Poly-DMAPS in the MD gel has, however, been bound by covalent crosslinking, resulting in the absence of UCST behavior in the MD gel (Fig. 5b). In contrast, the DM gel presented thermosensitive viscosity at a constant shear rate. More interestingly, compared to the UCST behavior of linear poly-DMAPS-only solution (no chemical-crosslinking) shown in Fig. 2, the bulk DM gel presented a steady reduction in rheological strength when the temperature increased from $25^{\circ} \mathrm{C}$ to $60^{\circ} \mathrm{C}$. This continuous gradual decrease in viscosity might be attributed to the chemically crosslinked poly-MPC matrix. Although the aggregated polyDMAPS can be untangled once the temperature is above the UCST, poly-DMAPS is still confined in the hydrogel matrix. As shown in Fig. 5c, photos of the DM gel reveal a noticeable difference in transparency at low and high temperatures, but even at $55^{\circ} \mathrm{C}$ there are still some thin but opaque reticulation patterns, probably caused by chemically-bound poly-DMAPS.

However, the mechanical strength of the DM gel is weaker than the random gel, which is likely related to the inhomogeneity of its UCST component during the gelation process (Fig. 4c). We hypothesized that poly-DMAPS, as the free polymer, was immersed in the second polymeric matrix yet formed UCST aggregations at room temperature, reducing the interactions with MPC side chains during the UV-initiated polymerization. Given the weaker mechanical strength of the DM gel compared to the other DN gels, the thermo-reduced viscosity DM gel has high potential for future drug release applications, such as in heat-stimulated pain relief patches. ${ }^{29}$

Regarding mechanical failure, crack propagation is one of the important factors undermining the usability of hydrogels. ${ }^{30}$ 
a)

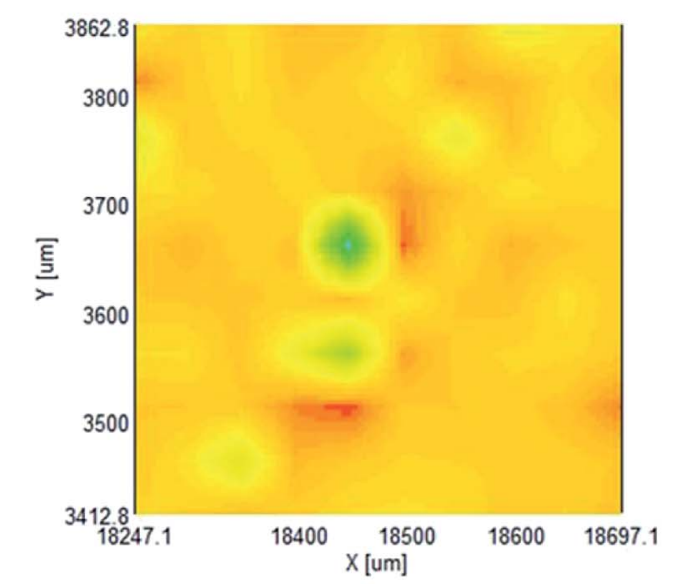

c)
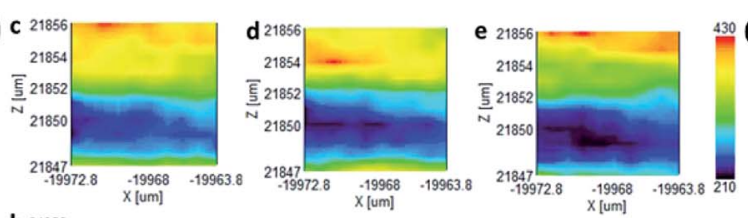

${ }^{430}$ d)
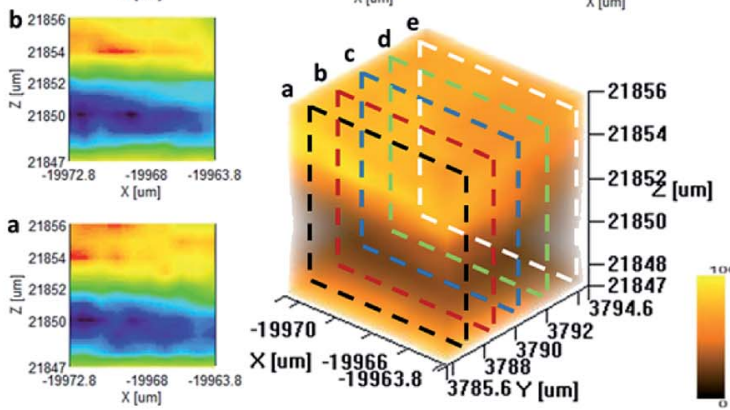

b)
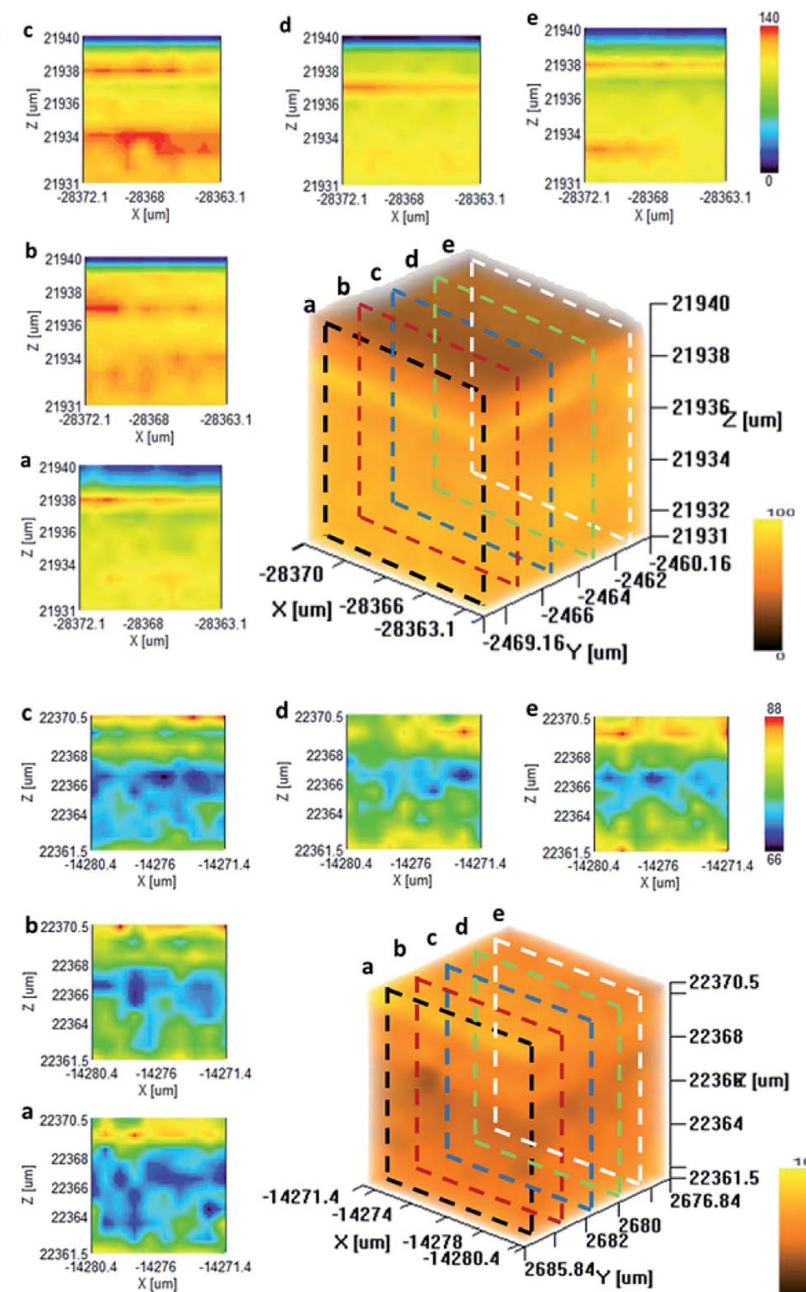

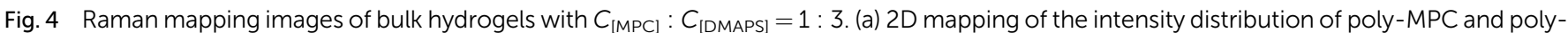

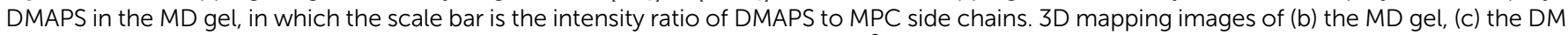

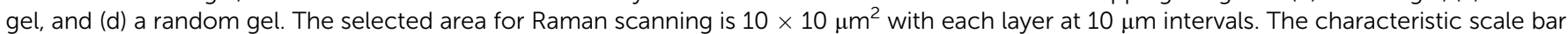
represents the intensity of the sulfonate group derived from poly-DMAPS.

To deeply understand the microstructures of the hydrogel, we investigated surface sections and cross-sections of the dualzwitterionic DN hydrogels via SEM imaging. As shown in Fig. 6, the MD gel was constructed of a dense and uniform interior network. On the contrary, a cross-section of the random gel showed clear porosity and elliptical pores, but these were still distributed homogeneously throughout the bulk gel, while the DM gel image revealed clear boundaries and uneven a)

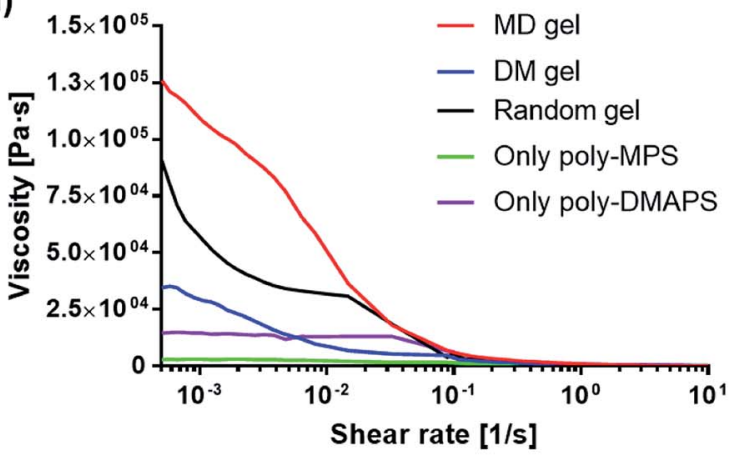

b)

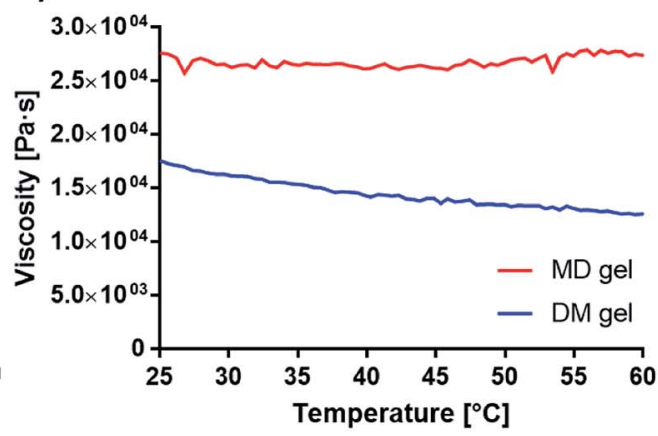

c)

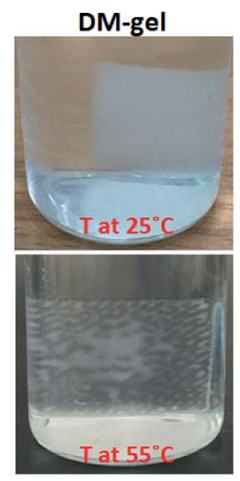

Fig. 5 Mechanical measurements of various zwitterionic hydrogels via a rheometer at an MPC : DMAPS molar ratio of $1: 3$. The hydrogels were studied (a) at different shear rates at a constant temperature of $25^{\circ} \mathrm{C}$ or (b) at different temperatures at the same shear rate $\left(0.01 \mathrm{~s}^{-1}\right)$ to investigate thermal sensitivity. (c) Photographs of the DM gel at room temperature or at $55^{\circ} \mathrm{C}$. 


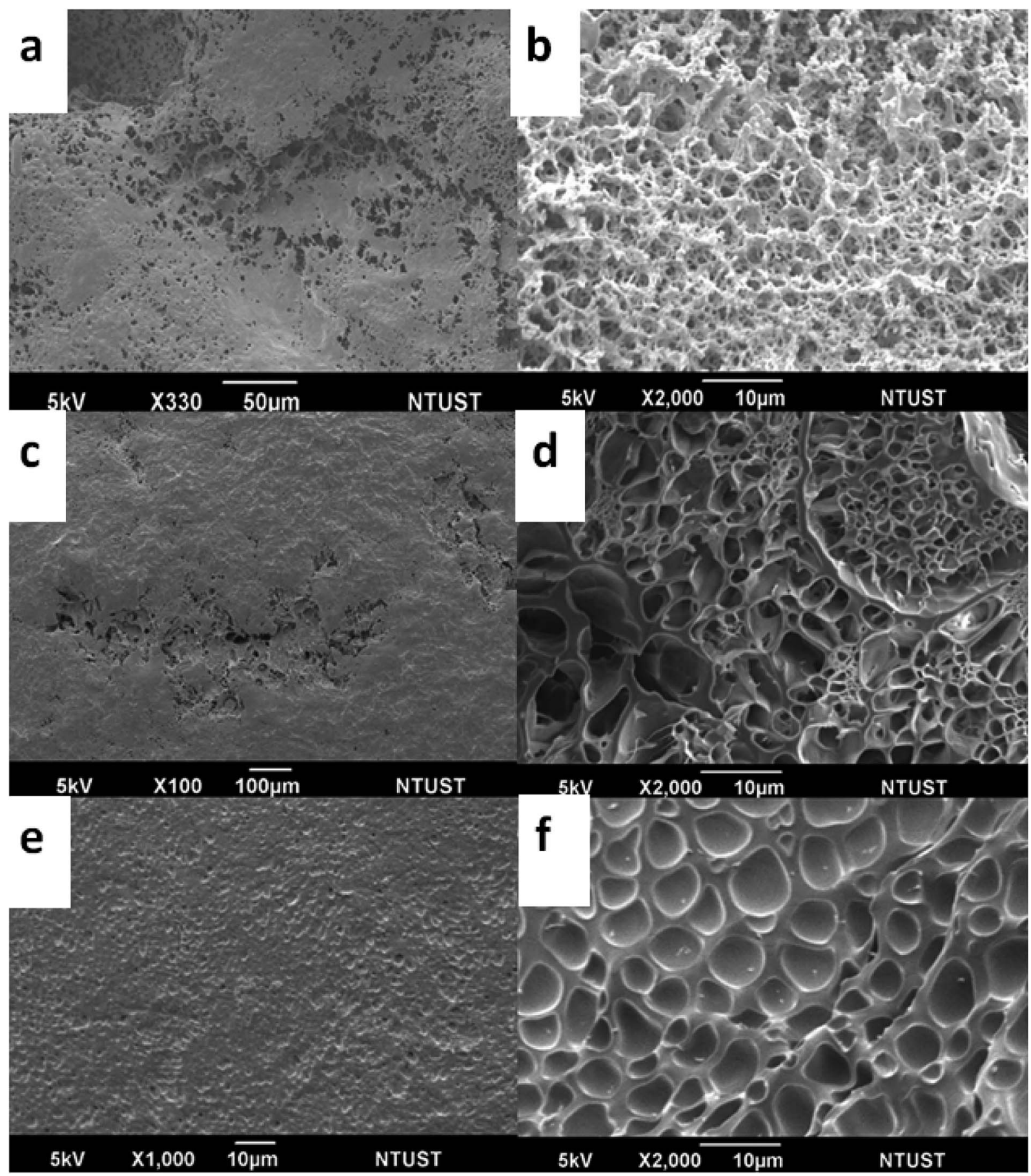

Fig. 6 Morphological images of different hydrogels on the surface and in cross-sections from SEM imaging. (a) The surface and (b) cross-section of the MD gel; (c) the surface and (d) cross-section of the DM gel; and (e) the surface and (f) cross-section of the random gel.

porosity across the cross-section. This also provides additional evidence to explain why the rheological viscosity of the hydrogels is in the order: MD gel > random gel > DM gel.

\section{Conclusions}

Zwitterionic hydrogels with promising properties, such as antifouling functionality, can be currently used in implanted devices or wound patches. However, their weak mechanical properties are still an unsolved issue for these bio-applications. Herein, hydrogels have been successfully prepared with an interpenetrating double network via the mixing of two zwitterionic polymers, poly-MPC and poly-DMAPS, to improve mechanical strength. The MD gel presented DN networkboosted mechanical strength and a homogeneous microstructure but lacked a thermosensitive response. On the other hand, unbound UCST poly-DMAPS in the DM hydrogel demonstrated a reduction in viscosity as the temperature increased. Although 
poly-DMAPS immersed in the DM gel lost its clear and instantaneous thermo-response, the DN network still improves the mechanical strength of the DM gel and promotes further applications, such as thermosensitive steady drug release via a warm compress for wound healing care or pain patches.

\section{Conflicts of interest}

All authors have seen and approved the revised manuscript and have no conflicts of interest concerning this publication.

\section{Acknowledgements}

The authors would like to thank the Ministry of Science and Technology, Taiwan (MOST 105-2221-E-011-133-MY3, 105-2221E-011-151-MY3 and 108-2923-E-011-005-MY3) for providing financial support.

\section{References}

1 E. Caló and V. V. Khutoryanskiy, Eur. Polym. J., 2015, 65, 252267.

2 L. Zhang, Z. Cao, T. Bai, L. Carr, J.-R. Ella-Menye, C. Irvin, B. D. Ratner and S. Jiang, Nat. Biotechnol., 2013, 31, 553.

3 K.-T. Huang, Y.-L. Fang, P.-S. Hsieh, C.-C. Li, N.-T. Dai and C.-J. Huang, J. Mater. Chem. B, 2016, 4, 4206-4215.

4 Y. Zhu, J. Zhang, J. Yang, C. Pan, T. Xu and L. Zhang, J. Mater. Chem. B, 2016, 4, 5105-5111.

5 A. M. S. Costa and J. F. Mano, Eur. Polym. J., 2015, 72, 344364.

6 L. Tang, W. Liu and G. Liu, Adv. Mater., 2010, 22, 2652-2656. 7 S. Y. Zheng, H. Ding, J. Qian, J. Yin, Z. L. Wu, Y. Song and Q. Zheng, Macromolecules, 2016, 49, 9637-9646.

8 J. Fang, A. Mehlich, N. Koga, J. Huang, R. Koga, X. Gao, C. Hu, C. Jin, M. Rief, J. Kast, D. Baker and H. Li, Nat. Commun., 2013, 4, 2974.

9 J. P. Gong, Y. Katsuyama, T. Kurokawa and Y. Osada, Adv. Mater., 2003, 15, 1155-1158.

10 T. Nakajima, H. Furukawa, Y. Tanaka, T. Kurokawa, Y. Osada and J. P. Gong, Macromolecules, 2009, 42, 2184-2189.

11 J. P. Gong, Soft Matter, 2010, 6, 2583-2590.
12 A. B. Ihsan, T. L. Sun, S. Kuroda, M. A. Haque, T. Kurokawa, T. Nakajima and J. P. Gong, J. Mater. Chem. B, 2013, 1, 45554562.

13 J. P. Gong, Y. Katsuyama, T. Kurokawa and Y. Osada, Adv. Mater., 2003, 15, 1155-1158.

14 S. Xiao, Y. Yang, M. Zhong, H. Chen, Y. Zhang, J. Yang and J. Zheng, ACS Appl. Mater. Interfaces, 2017, 9, 20843-20851.

15 H. Yin, T. Akasaki, T. L. Sun, T. Nakajima, T. Kurokawa, T. Nonoyama, T. Taira, Y. Saruwatari and J. P. Gong, J. Mater. Chem. B, 2013, 1, 3685-3693.

16 T. Ueda, H. Oshida, K. Kurita, K. Ishihara and N. Nakabayashi, Polym. J., 1992, 24, 1259.

17 N. Nakabayashi and Y. Iwasaki, Bio-Med. Mater. Eng., 2004, 14, 345-354.

18 K. Hirota, K. Murakami, K. Nemoto and Y. Miyake, FEMS Microbiol. Lett., 2005, 248, 37-45.

19 O. Azzaroni, A. A. Brown and W. T. Huck, Angew. Chem., Int. Ed., 2006, 45, 1770-1774.

20 M. Tian, J. Wang, E. Zhang, J. Li, C. Duan and F. Yao, Langmuir, 2013, 29, 8076-8085.

21 Q. Shao, L. Mi, X. Han, T. Bai, S. Liu, Y. Li and S. Jiang, J. Phys. Chem. B, 2014, 118, 6956-6962.

22 H. M. L. Lambermont-Thijs, H. P. C. v. Kuringen, J. P. W. v. d. Put, U. S. Schubert and R. Hoogenboom, Polymers, 2010, 2, 188-189.

23 J. S. Higgins, J. E. G. Lipson and R. P. White, Philos. Trans. $R$. Soc., A, 2010, 368, 1009-1025.

24 D. Ostrovskii, A. Brodin and L. Torell, Solid State Ionics, 1996, 85, 323-327.

25 Y. Koyama, S. Toda and Y. Kyogoku, Chem. Phys. Lipids, 1977, 19, 74-92.

26 Y. Fan and C. J. Cornelius, J. Mater. Sci., 2013, 48, 1153-1161.

27 K. C. Gordon and C. M. McGoverin, Int. J. Pharm., 2011, 417, 151-162.

28 Z. Zhang, T. Chao and S. Jiang, J. Phys. Chem. B, 2008, 112, 5327-5332.

29 H. He, Z. Xiao, Y. Zhou, A. Chen, X. Xuan, Y. Li, X. Guo, J. Zheng, J. Xiao and J. Wu, J. Mater. Chem. B, 2019, 7, 1697-1707.

30 Y. Tanaka, K. Fukao and Y. Miyamoto, Eur. Phys. J. E: Soft Matter Biol. Phys., 2000, 3, 395-401. 